AJCHE 2011, Vol. 11, No. 1, 1 - 7

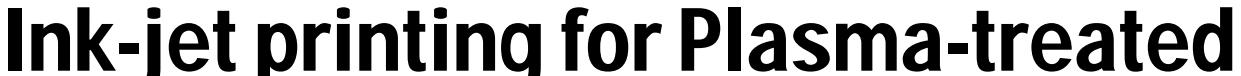

:DNU

\title{
C.W. Kan
}

C.W.M. Yuen

W.Y. Tsoi

C.K. Chan

Institute of Textiles and Clothing, the Hong Kong Polytechnic University,

Hung Hom, Kowloon, Hong Kong, China

*e-mail: tccwk@inet.polyu.edu.hk

Currently, sodium alginate is commonly used for preparing the printing medium for digital ink-jet printing due to its ready solubility and excellent stability even at high-temperature fixation treatments. Similar to sodium alginate, chitosan is also widely used as novel biomaterial. As a natural polymer, sodium alginate and chitosan are biocompatible, biodegradable and non-toxic. Therefore, chitosan may be used as a chemical for preparing printing medium for digital ink-jet printing for cotton fabric. Recently, low temperature plasma (LTP) treatment has proved to be an effective pretreatment method for the improving coating process by altering the surface properties of the material without much changing of the bulk properties. Thus, the aim of this study was to investigate the possibility of applying LTP treatment as pretreatment process to enhance the coating of printing medium, i.e. sodium alginate and sodium alginate/chitosan mixture, so as to improve the final properties of the digital ink-jet printed cotton fabric.

Keyword: low temperature plasma, cotton, surface, chitosan

\section{INTRODUCTION}

The application of digital ink-jet printing in the textile market was increased recently because this technique offers production benefits [1, 2]. In conventional textile printing, dyes are applied along with the printing chemicals in the form of a printing paste. However, due to the requirements of ink purity and specific conductivity for the digital ink-jet printing $[3,4]$, none of the conventional printing chemicals such as alkali, urea and sodium alginate can be directly incorporated into the ink formulation and therefore a printing medium should be prepared for textile digital ink-jet printing. Currently, sodium alginate is an important chemical for preparing the printing medium for digital ink-jet printing due to its ready solubility and excellent stability even after high-temperature fixation treatments. Similar to sodium alginate, chitosan is also widely used as novel biomaterial. As a natural polymer, sodium alginate and chitosan are biocompatible, biodegradable and non-toxic [5]. Previous research reported that chitosan could be applied to different textile areas for improving the colour yield, colour fastness and 
anti-bacterial function [5]. Therefore, chitosan may be used as a chemical for preparing the printing medium for digital ink-jet printing for cotton fabric.

For the digital ink-jet printing process for cotton fabric, the printing medium is coated on the fabric surface to facilitate the digital ink-jet printing. As a result, the effectiveness of coating the printing medium on the fabric will affect the final properties of digital ink-jet printed cotton fabric. Recently, low temperature plasma (LTP) treatment has proved to be an effective pretreatment method for the improving coating process by altering the material surface properties without much changing the bulk properties $[6,7]$. Thus, the aim of this study is to investigate the effectiveness of applying LTP treatment as pretreatment process to enhance the different coatings of printing medium with sodium alginate and sodium alginate/chitosan mixture so as to improve the final properties of the digital ink-jet printed cotton fabric.

\section{EXPERIMENTAL}

\section{Fabric Preparation and LTP Pretreatment}

$100 \%$ singed, desized, scoured and bleached cotton plain weave fabric. A glow discharge generator (Showa Co., Ltd., Japan) was used for the LTP pretreatment of the cotton fabrics. The glow discharge generator was a radio-frequency etching system operating at $13.56 \mathrm{MHz}$. Oxygen was fed to the system with a flow of 20 $\mathrm{ml} /$ minute. The discharge power and system pressure were set at $80 \mathrm{~W}$ and $10 \mathrm{~Pa}$, respectively. The duration of LTP pretreatment was 1, 2, 5, 15, 30 and 60 minutes.

\section{Preparation of Printing Medium with Sodium Alginate}

A sodium alginate stock solution was prepared by dissolving $50 \mathrm{~g}$ sodium alginate in $950 \mathrm{ml}$ of deionised water. The amount of sodium alginate used in the printing medium was measured directly from the sodium alginate stock solution. The printing medium was prepared by mixing $150 \mathrm{~g}$ sodium alginate, $8 \mathrm{~g}$ sodium bicarbonate, $10 \mathrm{~g}$ urea and finally making up to a weight of $400 \mathrm{~g}$ with deionised water [8]. The printing medium was well stirred for half an hour by means of an ultrasonic stirrer and was stored for 24 hours before being padded on the fabrics.

\section{Preparation of Printing Medium with Sodium Alginate/Chitosan Mixture}

A stock chitosan solution was prepared by dissolving $5 \mathrm{~g}$ chitosan completely into $500 \mathrm{ml}$ of $10 \%$ acetic acid with constant stirring. The stock solution was then filtered to remove any suspended impurities. The amount of chitosan used for preparing the printing medium was measured directly from the stock chitosan solution. The printing medium containing sodium alginate and chitosan was then prepared by mixing $150 \mathrm{~g}$ stock sodium alginate, $10 \mathrm{~g}$ chitosan solution, $8 \mathrm{~g}$ sodium bicarbonate, $10 \mathrm{~g}$ urea and finally making up to a weight of $400 \mathrm{~g}$ with deionised water. The printing medium was well stirred for half an hour by means of an ultrasonic stirrer and was stored for 24 hours before being padded on the fabrics.

\section{Fabric Pretreatment with Printing Medium}

The well mixed printing medium was padded onto the cotton fabric using a padding machine with an even pressure of $2.6 \mathrm{~kg} / \mathrm{m}^{2}$ and a constant padding speed of $2.5 \mathrm{rpm}$ until a pick-up of $80 \%$ was achieved. The treated fabrics were dried in an oven at $80^{\circ} \mathrm{C}$ and then conditioned at $20 \pm 2^{\circ} \mathrm{C}$ with relative humidity of $65 \pm 2 \%$ for 24 hours 
before digital ink-jet printing.

\section{Printing Procedure}

A digital ink-jet printer with piezoelectric drop-on-demand print head (Mimaki Tx2-1600, Mimaki Engineering Co., Ltd., Japan) was used and a commercially available reactive ink of yellow colour with vinylsulphone reacting system was used without further purification [9]. A pattern of size $80 \mathrm{~mm} \times 80 \mathrm{~mm}$ with resolution of 360 dots per inch was generated for easy comparison. After printing, the fabrics were air-dried and then placed into a steamer. All the printed fabrics were aftertreated with superheated steam at $110^{\circ} \mathrm{C}$ for $5 \mathrm{~min}$. for colour fixation. The steamed fabric samples were finally washed in a $10 \mathrm{~g} / \mathrm{l}$ non-ionic detergent until all the unreacted dye and chemicals were removed from the fabric surface.

\section{Colour Yield Measurement}

The colour yield of the printed fabrics was measured with a Macbeth Colour Eye 7000A Spectrophotometer. The colour yield expressed as a $\mathrm{K} / \mathrm{S}$ value in the range of wavelength from $400 \mathrm{~nm}$ to $700 \mathrm{~nm}$ according to Equation (1). The higher the $\mathrm{K} / \mathrm{S}$ value, the more will be the dye-uptake, resulting in better colour yield.

$\mathrm{K} / \mathrm{S}=(1-\mathrm{R})^{2} / 2 \mathrm{R}$

where $\mathrm{K}=$ absorption coefficient, depending on the concentration of colorant; $\mathrm{S}=$ scattering coefficient, caused by the dyed substrate; and $R=$ reflectance of the coloured sample

\section{Colour Fastness}

The colour fastness of the digital ink-jet printed fabrics was assessed by AATCC Test Method 61 (colour fastness to laundering) and AATCC Test Method 8 (colour fastness to crocking).

\section{Outline Sharpness Measurement}

In order to compare the outline sharpness of the prints, the widths of the printed pattern in both warp and weft directions were measured using an optical light microscope (Nikon Optiplot-pol, USA) with a magnification of 400 .

\section{Anti-bacterial Effect}

The anti-bacterial effects of the digital ink-jet printed fabrics were quantitatively evaluated by AATCC Test Method 100.

\section{RESULTS AND DISCUSSION \\ Colour Yield Measurement}

Figure 1 shows that the colour yield of the LTP pretreated fabrics with different pretreatment times was higher than the untreated fabric. The colour yield increased gradually with LTP pretreatment time and reached the maximum at 2 minutes of pretreatment time. Further increase of LTP pretreatment time did not give further enhancement of the colour yield but produced a gradual colour yield reduction instead. Urea is used in the printing medium and it can swell the cotton fibres during steaming process, especially with superheated steaming, so that dye can penetrate into them rapidly. As urea holds water very strongly, so a mixture of urea and water can provide the solvent required for the reaction to occur within the fibres. Hence, urea acts as a solvent for reactive dye because it functions like a moisture-absorbing agent in the printing medium to increase moisture regain during steaming, thereby accelerating the migration of the dye from the printing medium into the cotton fibres. Since reactive dye with vinylsulphone structure was used in this study, it had a tendency to be deactivated in the presence of urea due to the thermal decomposition. This would result in the conversion of the 
vinylsulphone dye to an inactive aminoethylsulphone rather than in a direct reaction between the dye and fibre leading to a decrease in the colour yield of the LTP pretreated fabrics. On the other hand, with a longer LTP exposure time using oxygen gas, the amount of hydrophilic groups such as $-\mathrm{CO}-,-\mathrm{C}=\mathrm{O}-$ and $-\mathrm{COOH}$ on the cellulosic fibre surface would increase correspondingly as indicated by surface analysis results. The increased amount of hydrophilic groups coupled with the urea present in the printing medium could hold more moisture during the steaming process. As a result, hydrolysis of the reactive dye might occur and reduce the colour yield. For the printing medium containing sodium alginate/chitosan mixture, Figure 1 demonstrates that about $85 \%$ of the final colour yield was achieved when compared with the printing medium with sodium alginate alone. The lowered colour yield may be due to the neutralization effect occurred during the mixing of chitosan solution and sodium bicarbonate in preparing the printing medium. The neutralization effect might thus reduce the effectiveness of the printing medium during the digital ink-jet printing process.

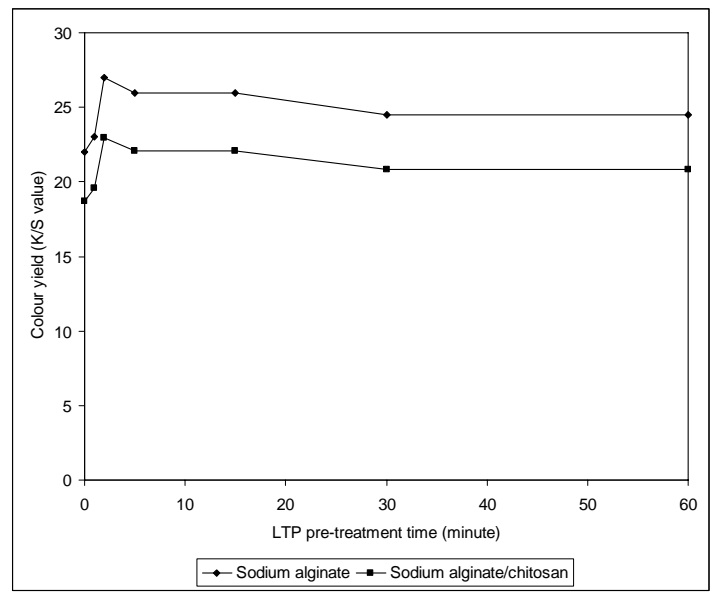

Figure 1. $\mathrm{K} / \mathrm{S}$ values of digital ink-jet printed cotton fabric pretreated with different LTP pretreatment time and printing medium

\section{Colour Fastness Tests}

Table 1. Result of colour fastness to crocking and washing

\begin{tabular}{|c|c|c|c|c|c|}
\hline \multicolumn{2}{|c|}{ Fabric Samples } & \multicolumn{2}{|c|}{ Crocking } & \multicolumn{2}{|c|}{ Washing } \\
\hline & & Dry & Wet & $\mathrm{CS}^{*}$ & $\mathrm{~S}^{* *}$ \\
\hline \multirow{7}{*}{$\begin{array}{l}\text { Fabrics } \\
\text { coated } \\
\text { with } \\
\text { sodium } \\
\text { alginate } \\
\text { printing } \\
\text { medium }\end{array}$} & $0 \mathrm{~min}$. & 4.5 & 4 & 4 & 4.5 \\
\hline & $1 \mathrm{~min}$. & 4.5 & 3.5 & 4 & 4.5 \\
\hline & $2 \mathrm{~min}$. & 4.5 & 3.5 & 4 & 4.5 \\
\hline & $5 \mathrm{~min}$. & 4.5 & 3.5 & 4 & 4.5 \\
\hline & $15 \mathrm{~min}$. & 4.5 & 3.5 & 4 & 4.5 \\
\hline & $30 \mathrm{~min}$. & 4.5 & 3.5 & 4 & 4.5 \\
\hline & $60 \mathrm{~min}$. & 3.5 & 2.5 & 4 & 4.5 \\
\hline \multirow{7}{*}{$\begin{array}{l}\text { Fabrics } \\
\text { coated } \\
\text { with } \\
\text { sodium } \\
\text { alginate / } \\
\text { chitosan } \\
\text { mixture } \\
\text { printing } \\
\text { medium }\end{array}$} & $0 \mathrm{~min}$. & 5 & 4.5 & 4 & 5 \\
\hline & $1 \mathrm{~min}$. & 5 & 4 & 4.5 & 4.5 \\
\hline & $2 \mathrm{~min}$. & 5 & 4 & 4.5 & 4.5 \\
\hline & $5 \mathrm{~min}$. & 5 & 4 & 4.5 & 4.5 \\
\hline & 15 min. & 5 & 4 & 4.5 & 4.5 \\
\hline & $30 \mathrm{~min}$. & 5 & 4 & 4.5 & 4.5 \\
\hline & $60 \mathrm{~min}$. & 4.5 & 3.5 & 4.5 & 4.5 \\
\hline
\end{tabular}

* Colour change

* Staining - the fibre components in the multi-fibre fabric included wool, acrylic, polyester, nylon, cotton and acetate. The numbers indicate the grade scale rating in which the higher the grade scale rating, the better will be the colour fastness

From Table 1, it could be seen that the overall results of all samples on the dry colour fastness to crocking test were good (grade 4-5) except those samples with 60 minutes of LTP pretreatment had comparatively lower grade. As for the wet crocking test, the samples got relatively lower grades when compared with the dry crocking. In particular for the sample treated for 60 minutes LTP pretreatment with sodium alginate printing medium alone, it scored grade 2.5 only. The reasons of poor crocking fastness of 60 minutes of LTP pretreated samples might due to long exposure to LTP pretreatment causing surface damage on the fabric samples. For the colour fastness to 
washing, the overall results of colour change and staining were 4 or above which is at an acceptable level.

When the types of printing medium were compared, the printing medium with sodium alginate alone has a lower rating than the printing medium with sodium alginate/chitosan mixture. The improvement might be due to the introduction of primary amino groups into the cellulosic fibre structure. Most probably after these groups were deposited in the crevices between the fibres, they might impart a cationic surface which attracted the oppositely charged reactive dye anions. Although the hydrolysed dyes may be attached to chitosan molecules without fixation and the chitosan in cotton fabric can be easily removed from the fabric with repeated laundering, the improved results of colour fastness indicated that the reactive dyes in the ink are held firmly on the cotton fabric when compared with the untreated fabric. This reveals that more reactive dyes are absorbed by the cotton fabric and the improved absorption may be due to the enhanced physical and chemical attractions between the reactive dyes and the chitosan-treated cotton fabric.

\section{Outline Sharpness Measurement}

The outline sharpness of the digital ink-jet printed pattern was measured by the optical analysis method with the results shown in Table 2. Obviously, the digital ink-jet printed patterns in the warp direction were slightly wider than those in the weft direction for both untreated and LTP pretreated fabrics. This might be due to the differential wicking effect caused by the warp and weft yarns. When comparing the width of the printed patterns, the patterns printed on the LTP pretreated cotton fabrics were narrower than on the untreated fabric in both warp and weft directions. This could be attributed to the reduced spreading of the printed reactive inks as a result of the strong fibre and dye attraction because of the formation of covalent bonds between the hydroxyl groups of fibre and the reacting system of the reactive dye present in the printing ink. Consequently, the LTP pretreatment on cotton fabric could enhance the outline sharpness of the ink-jet prints. In addition, when chitosan was added in the printing medium, the patterns are narrower which might be due to the introduction of cationic groups $\left(-\mathrm{NH}_{3}{ }^{+}\right)$presence in the chitosan into the cotton fabric resulting in a strong ionic attraction between cationic cotton and anionic reactive inks.

Table 2. Outline sharpness of specimens with different LTP pretreatment time

\begin{tabular}{|c|c|c|c|c|}
\hline \multirow[t]{3}{*}{ Sample } & \multicolumn{4}{|c|}{ Sharpness with different printing medium } \\
\hline & \multicolumn{2}{|c|}{ Warp $(\mathrm{mm})^{*}$} & \multicolumn{2}{|c|}{ Weft $(\mathrm{mm})^{*}$} \\
\hline & $\begin{array}{l}\text { Sodium } \\
\text { alginate }\end{array}$ & $\begin{array}{l}\text { Sodium } \\
\text { alginate / } \\
\text { chitosan } \\
\text { mixture }\end{array}$ & $\begin{array}{l}\text { Sodium } \\
\text { alginate }\end{array}$ & $\begin{array}{l}\text { Sodium } \\
\text { alginate / } \\
\text { chitosan } \\
\text { mixture }\end{array}$ \\
\hline Control & 82 & 82 & 81 & 81 \\
\hline $1 \mathrm{~min}$. & 81.5 & 81 & 81 & 81 \\
\hline $2 \mathrm{~min}$. & 81.5 & 81 & 81 & 80.5 \\
\hline $5 \mathrm{~min}$. & 81.5 & 81 & 81 & 80.5 \\
\hline $15 \mathrm{~min}$. & 81 & 80.5 & 80.5 & 80 \\
\hline $30 \mathrm{~min}$. & 81 & 80.5 & 80.5 & 80 \\
\hline $60 \mathrm{~min}$. & 81 & 80.5 & 80.5 & 80 \\
\hline \multicolumn{5}{|c|}{$\begin{array}{l}\text { * The widths of the printed pattern were } \\
\text { measured in } \mathrm{mm} \text { in both warp and weft } \\
\text { directions. If the number is greater / smaller } \\
\text { than the control sample, the wider / narrower } \\
\text { will be the width of the printed pattern when } \\
\text { compared with the control sample }\end{array}$} \\
\hline
\end{tabular}

\section{Anti-bacterial Effect}

AATCC Test Method 100 was used to determine the anti-bacterial activity of the digital ink-jet printed fabrics with different printing medium. Table 3 shows the 
bacterial concentration of Staphylococcus aureus bacteria with respect to the digital ink-jet printed fabrics with different printing medium, under the influence of 2 minutes LTP pretreatment time, during 0 hours and 48 hours.

Table 3. Anti-bacterial evaluation of

\begin{tabular}{lccc}
\multicolumn{1}{c}{ specimens with different printing medium } \\
\cline { 2 - 4 } & 0 hours 48 hours & $\begin{array}{c}\% \text { of } \\
\text { bacterial } \\
\text { removal } \\
(-\mathrm{-ve}) / \\
\text { increase } \\
(+\mathrm{ve})\end{array}$ \\
\hline $\begin{array}{l}\text { Control (2 } \\
\text { minutes }\end{array}$ & $1.28 \times 10^{8}$ & $2.22 \times 10^{8}$ & $+172.4 \%$ \\
$\begin{array}{l}\text { LTP } \\
\text { pre-treated } \\
\text { fabric) }\end{array}$ & & & \\
$\begin{array}{l}\text { Sodium } \\
\text { alginate } \\
\text { Sodium } \\
\text { alginate / } \\
\text { chitosan } \\
\text { mixture }\end{array}$ & $2.39 \times 10^{8}$ & $6.90 \times 10^{7}$ & $-71.1 \%$ \\
& & & $-100 \%$ \\
\end{tabular}

The results in Table 3 indicated that the chitosan containing fabrics shows positive result of anti-bacterial effect, meaning that the bacteria could be completely killed by the chitosan present in the treated cotton fabric. Therefore, the chitosan treatment for cotton fabric was very effective to resist bacteria when compared with the control fabric. Although the control fabric containing sodium alginate alone had the function of anti-bacterial effect, its performance was not as good as the chitosan containing fabric. The control fabric did not exhibit any anti-bacterial effect as reflected by a large number of bacteria growth.

\section{CONCLUSION}

An attempt was conducted to pretreat cotton fabric with LTP pretreatment with various treatment duration followed by coating with printing medium containing (i) sodium alginate alone and (ii) sodium alginate/chitosan mixture for studying the influence of the colour yield with digital ink-jet printing. Experimental results showed that with 2 minutes of LTP pretreatment, maximum colour yield could be obtained significant for both types of printing medium but decreased at prolonged exposure times. As a whole, the sodium alginate/chitosan mixture printing medium achieved about $85 \%$ of the colour yield when compared with the printing medium containing sodium alginate alone. However, other studied properties such as colour fastness to crocking, colour fastness to washing, outline sharpness measurement and anti-bacterial evaluation indicated that sodium alginate/chitosan mixture printing medium gave a better result than printing medium containing sodium alginate alone.

\section{ACKNOWLEDGEMENT}

The work described in this paper was supported by a grant from the Research Grants Council of the Hong Kong Special Administrative Region, China (Project No. PolyU 5192/08E) and a research grant from the Hong Kong Polytechnic University.

\section{REFERENCES}

1) S. Gupta, Indian Journal of Fibre and Textile Research, 26(1-2) (2001) 156.

2) M. van Parys, Melliand Texilberichte / International Textile Reports (English Edition) 83(6) (2002) E96.

3) P.S.R. Choi, C.W.M. Yuen, S.K.A. Ku and 
CW Kan, CWM Yuen, WY Tsoi and CK Chan 7

C.W. Kan, Textile Asia, 35(2) (2005) 50.

4) S.O. Aston, J.R. Provost and H. Masselink, Journal of Society of Dyers and Colourists, 109(4) (1993) 147.

5) P.S.R. Choi, C.W.M. Yuen, S.K.A. Ku and C.W. Kan, Fibers and Polymers, 6(3) (2005) 229.

6) R. Shishoo, Journal of Coated Fabrics 26(2) (1996) 87.

7) S. Luo and W. van Ooij, Journal of
Adhesion Science and Technology, 16(13) (2002) 1715.

8) C.W.M. Yuen, S.K.A. Ku, P.S. Choi and C.W. Kan, Fibers and Polymers, 5(2) (2002) 117.

9) C.W.M. Yuen, S.K.A. Ku, P.S. Choi and C.W. Kan, Textile Research Journal, 75 (2005) 319. 